\title{
Clinical Assessment of Low Back Pain: A Neurosurgical Point of View
}

\author{
${ }^{1}$ Alessandro Landi, ${ }^{2}$ Giovanni Grasso, ${ }^{3}$ Davide Colistra, ${ }^{4}$ Angela Ambrosone, ${ }^{5}$ Roberto Delfini
}

\begin{abstract}
The diagnosis of low back pain (LBP) is complex and multifactorial, and a clear etiology responsible for the symptoms is often not detectable. Clinical history and neurological examination are essential for differential diagnosis, because they allow the identification of the specific pathology. The radiological investigation allows to confirm diagnosis, but the indication must always be correlated with clinical findings to not perform unnecessary diagnostic procedures. Management of LBP can involve many specialists - neurosurgeon, orthopedist, physiatrist, physiotherapist - everyone with different knowledge and experience because very often the cause is multifactorial. However, it is mandatory to clarify diagnosis of LBP; in this way, the clinical pathway is fundamental to understand pathogenesis and to determine appropriate treatment.
\end{abstract}

Keywords: Clinical diagnosis, Low back pain, Lumbar spine pathology, Neurological tests, Physical exam.

How to cite this article: Landi A, Grasso G, Colistra D, Ambrosone A, Delfini R. Clinical Assessment of Low Back Pain: A Neurosurgical Point of View. Int J Recent Surg Med Sci 2016;2(2):55-57.

Source of support: Nil

Conflict of interest: None

\section{INTRODUCTION}

Low back pain (LBP) is a chronic remitting pain without neurological deficits, ${ }^{1-3}$ which can affect people of all ages without gender differences. Management of LBP can involve many specialists - neurosurgeon, orthopedist, physiatrist, physiotherapist - everyone with different knowledge and experience because very often the cause is multifactorial. However, it is mandatory to clarify diagnosis of LBP, since it is the first cause of outpatient medical care in Western countries, and misdiagnosis may lead to an useless or dangerous treatment. So, clinical diagnosis is fundamental to understand pathogenesis and to determine appropriate treatment.

\footnotetext{
${ }^{1}$ Researcher, ${ }^{2}$ Professor, ${ }^{3}$ Resident, ${ }^{4}$ Resident, ${ }^{5}$ Professor and Chief

1,3-5 Department of Neurology and Psychiatry, Division of Neurosurgery A, "Sapienza" University of Rome, Italy

${ }^{2}$ Department of Biomedicine and Clinical Neurosciences Neurosurgical Clinic, University of Palermo, Italy

Corresponding Author: Alessandro Landi, Department of Neurology and Psychiatry, Division of Neurosurgery A "Sapienza" University of Rome, Italy, Phone: +390649979105 e-mail: dott.alessandro.landi@gmail.com
}

\section{CLASSIFICATION}

Low back pain can be classified as follows ${ }^{4-6}$ :

- Physiopathological characteristics: Based on physiopathology, we can distinguish three types of LBP: "nociceptive LBP" in which the pain is caused by a stimulus in the neuronal pathway as response to "nociceptive pulses" derived from sport injuries, disk degeneration, facet joint arthropathy, disk herniations; "mixed LBP" in which the pain is generated by both primary and secondary injuries; "neuropathic LBP" in which the pain is caused by primary injury of the nerve.

- Pathology that generates the pain: "mechanical LBP": the pain is caused by degenerative and traumatic diseases with vertebral instability; "nonmechanical LBP": The pain is caused by neoplastic, infectious, and inflammatory diseases; "LBP caused by visceral diseases": Referred pain caused by kidney pathology, aortic aneurysms, gastrointestinal diseases.

- Clinical symptoms: Low back pain that has an onset with pain without deficit and LBP that shows up with deficit without pain.

The diagnosis of LBP must provide an accurate clinical history, a careful clinical examination, and specific and targeted technical investigations. The clinical history is fundamental to drive the diagnostic hypotheses since it allows to make a first differential diagnosis.

\section{CLINICAL ASPECTS OF LBP}

Based on the clinical classification of LBP, we must immediately distinguish the so-called red flags, namely the alarm signs of serious underlying diseases. Major red flags are night pain or pain at rest, fever associated with drug resistance and weight loss, joint stiffness in the morning. These anamnestic data are all indices of gravity of the pathology responsible for LBP. It is absolutely critical to evaluate the presence of such symptoms in order to properly direct the diagnostic process; in the light of this, clinical characteristics are crucial for a correct diagnosis:

Low back pain associated with fever and weight loss: In this case, the main suspect is an infectious disease. The most frequent infectious diseases that cause LBP are osteomyelitis, discitis, abscess, and meningitis. The clinical status associated with infectious disease 
presents typical characteristics, such as fever, shivers, nocturnal pain, and weight loss. It has a slow progression, worsens with movement, and is not drug responsive. In this case, specific diagnostic exams are mandatory to make diagnosis, especially laboratory tests. Extremely useful is a biopsy, especially computed tomography (CT) scan guided, because it allows the characterization of infection to properly start a targeted antibiotic therapy.

Low back pain associated with nocturnal and rest pain: In this case, the main suspect is a neoplastic disease. Obviously, neoplastic lesions that may be responsible for LBP vary from benign lesion (meningioma, schwannoma, etc.) to malignant or metastatic tumors. The clinical status in this case is quite specific and is characterized by LBP that often precedes the onset of neurological deficits. It can be associated with fever, shivers, weight loss, etc. The progression of symptoms is often very fast. When there are vertebral metastasis, pain often worsens with supine position. The onset of neurological deficits, such as paresis/plegia or sfinteric deficits, is often present in a late stage of disease.

Low back pain with joint stiffness in the morning: The main suspect in this case is a rheumatic disease, such as rheumatoid arthritis, ankylosing spondylitis, and seronegative spondyloarthritis. The clinical status is characterized by LBP that arises insidiously at a young age (often before 40 years) associated with a joint stiffness in the morning longer than 60 minutes, which improves with movement. We can find nocturnal pain, loss of movement in all three spatial planes, improvement in the pain with movement, and rarely neurological deficits. Very often, the sacroiliac joint is involved; when the sacroiliac joint is involved, above all in rheumatoid arthritis and ankylosing spondylitis, there is tenderness and acute pain associated with sacroiliac joint palpation. ${ }^{7}$ It is important to differentiate coxarthrosis, sacroiliitis, and L1-L2-L3 radiculopathy, where symptoms are extremely similar. In sacroiliitis, the pain is above all symmetrical and bilateral; it increases with resting and regresses with walking and the sacroiliac joint tests are positive. ${ }^{8}$

Low back pain associated with movement: The main cause is spinal degeneration. ${ }^{9}$ In this case, the onset of symptoms is acute or chronic, pain worsens with movement, and there is no nocturnal pain. It is very often associated with the assumption of antalgic abnormal postures. It is possible to find neurological deficits. The most common differential diagnosis is with piriformis syndrome. In this case, the muscle can compress the sciatic nerve, which may cause a deep pain in the gluteal region. It increases to palpation and it could be associated with a sciatica, which worsens with race and with the sitting position.
Acute and localized LBP with possible deficits: The main cause is traumatic disease due to high-energy impact. Also a low-energy trauma can cause fracture if the bone is degenerated by some diseases like osteoporosis, osteomalacia, Paget's syndrome, etc. The onset of pain is acute and it is often associated with acute severe neurological deficits. There is sometimes a sphincter involvement.

Low back pain with abnormal characteristics: In this case, we have to consider the possibility that the pain can be psychogenic. For this reason, we must always assess the positivity of Waddell's signs that precisely address the psychogenic nature of the $\mathrm{LBP}^{10}$ :

- Superficial and diffuse tenderness and/or nonanatomic tenderness

- Regional weakness or sensory changes which deviate from accepted neuroanatomy

- Movements which produce pain, without actually causing that movement

- Positive tests are rechecked when the patient's attention is distracted

In this case, clinical examination is extremely important in order to properly characterize the LBP.

\section{MANAGEMENT OF LBP}

Medical history and clinical history provide the primary data to make an initial differential diagnosis of LBP. Obviously, this diagnosis must be confirmed by neurological and physical examination through careful inspection and palpation of the back, assessment of posture and of a range of global and segmental motion, functional assessment of the neighboring joints, signs of root irritation with assessment of reflexes and of sensorimotor integrity. If we suspect a neoplastic lesion, we should associate a general physical examination. Obviously, neurological examination must be followed by specific radiological investigations in order to definitely confirm the pathology responsible for the LBP.

\section{CONCLUSION}

The diagnosis of LBP is complex and multifactorial, and a clear etiology responsible for the symptoms is often not detectable. Clinical history and neurological examination are essential for differential diagnosis, because they allow the identification of the specific pathology. The radiological investigation allows to confirm diagnosis, but the indication must always be correlated with clinical findings, so as not to perform unnecessary diagnostic procedures. The management of LBP is important for a multidisciplinary evaluation. Management of LBP can involve many specialists - neurosurgeon, orthopedist, physiatrist, physiotherapist - everyone with different knowledge and experience, which all play an equally important role. 


\section{REFERENCES}

1. Dillingham T. Evaluation and management of low back pain: an overview. State Art Rev 1995;9(3):559-574.

2. Nachemson A. Back pain: delimiting the problem in the next millennium. Int J Law Psychiatry 1999 Sep-Dec;22(5-6):473-490.

3. Wittenberg RH, Greskotter KR, Steffen R, Schoefeld BL. Is epidural injection treatment with hypertonic saline solution in intervertebral disk displacement useful? The effect of $\mathrm{NaCl}$ solution on intervertebral disk tissue. Z Orthop Ihre Grenzgeb 1990 Mar-Apr;128(2):223-226.

4. Amirdelfan K, McRoberts P, Deer TR. The differential diagnosis of low back pain: a primer on the evolving paradigm. Neuromodulation 2014 Oct;17 (Suppl 2):11-17.

5. O'Sullivan P. Diagnosis and classification of chronic low back pain disorders: maladaptive movement and motor control impairments as underlying mechanism. Man Ther 2005 Nov;10(4):242-255.
6. Allegri M, Montella S, Salici F, Valente A, Marchesini M, Compagnone C, Baciarello M, Manferdini ME, Fanelli G. Mechanisms of low back pain: a guide for diagnosis and therapy. 2016 Jun 28;5. F1000Res. Faculty Rev-1530. eCollection 2016.

7. Laslett $\mathrm{M}$, Williams $\mathrm{M}$. The reliability of selected pain provocation tests for sacro-iliac joint pathology. Spine 1994 Jun 1;19(11):1243-1249.

8. Landi A, Grasso G, Mancarella C, Delfini R. The impact of sacro-iliac joint dysfunction in the management of low back pain and failed back surgery syndrome. J Spine 2016;5: 321.

9. Goode AP, Carey TS, Jordan JM. Low back pain and lumbar spine osteoarthritis: how are they related? Curr Rheumatol Rep 2013 Feb;15(2):305.

10. Waddell G. Short academic biography with selected publications. Available from: mh-hannover.de. Archived from the original on May 2, 2014. Retrieved June 23, 2015. 